\title{
ANÅLOGO QUADRUPOLAR PARA A FASE NEMÁTICA
}

Cesar de 01 iveira Lobo

Departamento de Física. Centro de Ciências Naturais e Exatas. UFSM. Santa Maria, RS.

Vitor Hugo F. Santos

Departamento de Fisica. UFSC. Florianōpolis, SC.

RESUMO

Desenvolveu-se aqui um hamiltoniano completo, análogo ao modelo de Ising de spin 1, para a fase nemática de cristais líquidos, no qual este é considerado como um sistema em que as interaçôes predominantes săo de tipo quadrupolar e estão acopladas fracamente a um campo externo, elétrico ou magnético, uniforme e transverso.

SUMMARY

OLIVEIRA LOBO, C. da and SANTOS, V. H. F., Quadrupolar analogous for nematic phase. Ciência e Natura, 13: 7-9, 1991.

We obtained a full hamiltonian to the nematic phase of liquid crystals, whic his analog to the Ising model for a system of spin-1 particles. In this hamiltonian we considered that the main interations are quadripole-like and that they are wehkly coupled to an uniform and transverse electric or magnectic exter nal field.

\section{INTRODUÇÃO}

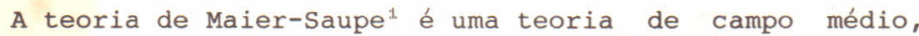
onde a interaçăo molecular dipolo-dipolo, responsável pela ordenação apresentada pelas moléculas de um cristal líquido, é substituída por um potencial efetivo função do ângulo $\theta$ que o ei xo das moléculas faz com a direçăo do vetor diretor $\vec{D}$. Em presença de um campo externo uniforme, elétrico ou magnético, aplicado na direção $z$. Lin-Lei ${ }^{2}$ obteve um hamiltoniano útil para a teoria de Maier-Saupe:

$$
H=-\frac{1}{2} \underset{i, j}{\Sigma} V_{i, j} P_{2}\left(\cos \theta_{i}\right) P_{2}\left(\cos \theta_{j}\right)
$$

onde $v_{i, j}$ é a constante de interaçăo efetiva, igual para todos os pares de moléculas. Posteriormente Tareeva ${ }^{3}$ propôs um análogo do modelo Ising ${ }^{4}$ par partículas com spin 1 e interaçăo quadrupolar, que se mostrou apropriado para descrever os cristais líquidos. Este hamiltoniano é dado por:

$$
H=-\frac{1}{2} \sum_{i, j}\left(3 s_{i}^{2}-2\right)\left(3 s_{i}^{2}-2\right)
$$

Mais recentemente, Santos e Lauck propuseram o mesmo modelo para sistemas com spin s qualquer:

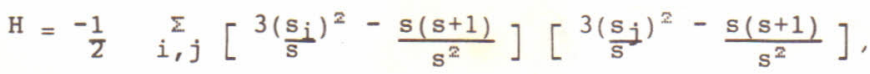


onde $s_{i}=s_{i} z$ é a componente $z$ do spin $\vec{s}$. Estes autores mostraram que este hamiltoniano tem a vantagem de recobrar $\circ$ modelo de Maier-Saupe para valores grandes de $s \quad(s \rightarrow \infty)$, e é útil para descrever a fase nemática de um cristal líquido.

TEORIA

Se definirmos os operadores quadrupolares $Q_{i}{ }^{\infty}=(\alpha=0$, $1,2,3,4)$, em termos dos operadores de spin como:

$$
\begin{aligned}
& Q_{i}{ }^{0}=\left(s_{i}{ }^{z}\right)^{2}-\frac{2}{3} \\
& Q_{i}{ }^{1}=\left(s_{i}{ }^{x}\right)^{2}-\left(s_{i}\right)^{2} \\
& Q_{i}{ }^{2}=s_{i}{ }^{X} s_{i} Y+s_{i} y_{s_{i}}{ }^{X} \\
& Q_{i}{ }^{3}=s_{i}{ }^{X} s_{i}{ }^{z}+s_{i}{ }^{z} s_{i}{ }^{X} \\
& Q_{i}{ }^{4}=s_{i}{ }^{Y} s_{i}{ }^{z}+s_{i}{ }^{z} s_{i}{ }^{Y}
\end{aligned}
$$

podemos escrever o hamiltoniano da equação (3), para $\mathbf{s}=1$, na forma:

$$
H=-9 \propto \sum_{i, j}\left[\left(\frac{S_{j}{ }^{z}}{s}\right)^{2}-\frac{2}{3}\right]\left[\left(\frac{S_{j}{ }^{z}}{s}\right)^{2}-\frac{2}{3}\right],
$$

ou seja,

$$
H=-\frac{1}{2} \sum_{i, j}^{\Sigma} V_{i, j} Q_{i}^{0} Q_{j}{ }^{\circ}
$$

o potencial de interação de um nemático com um campo externo fraco transverso $\overrightarrow{\mathrm{H}}=\mathrm{H} \hat{\mathrm{l}}$ é dado, neste caso, por:

$$
\mathrm{v}=-\frac{1}{2} \times \mathrm{H}^{2} \cos ^{2}(\theta \mathrm{x}),
$$

onde $x$ é a susceptibilidade do meio. É conveniente que uma constante $\mathrm{V}_{0}$ seja adicionada à equaçăo (6)

$$
\mathrm{V}_{0}=\frac{1}{6} \times \mathrm{H}^{2}
$$

que, assim, toma a forma:

$$
V=-\frac{1}{3} \times H_{X}^{2} P_{2}\left(\cos \theta_{X}\right)
$$

onde $P_{1}(\cos \theta)$ são os polinômios de Legendre. Agora, considerando que:

e que:

$$
P_{1}(\cos \theta)=\frac{4 \pi}{21+1} \sum_{m=-1}^{1} \gamma_{1}^{m}(\theta, \Phi)^{*} \quad \gamma_{1} m(\theta, \Phi)
$$

$$
\begin{aligned}
& \gamma_{z}{ }^{\circ}(\theta, \Phi)=-\frac{2}{5} \sqrt{5 / 4 \pi} \quad\left[\frac{3}{2} L_{z}{ }^{2}-\frac{1}{2} L(L+1)\right] \\
& \gamma_{2}^{ \pm 2}(\theta, \Phi)=-\frac{2}{5} \sqrt{15 / 32 \pi} L_{ \pm}{ }^{2}
\end{aligned}
$$


ficamos com:

$$
P_{2}(\cos \theta)=-\frac{2}{5}\left[\frac{3}{8}\left(L_{+}{ }^{2}+L_{-}{ }^{2}\right)-\frac{1}{2}\left[\frac{3}{2} L_{z^{2}}-\frac{1}{2}(L+1)\right]\right]
$$

No nosso caso os operadores de momento angular L correspondem aos spins $\mathbf{s}=1$ das moléculas. Com isso o potencial de interaçăo, representado na equação (7), toma a forma:

$$
\mathrm{v}=-\Omega \sum_{i}\left[Q_{i}{ }^{1}-Q_{i}{ }^{0}\right] \text {, }
$$

onde

$$
\Omega=-\frac{1}{10} \times \mathrm{H}_{x^{2}}
$$

e os $Q_{i}{ }^{\alpha}$ foram definidos acima na equaçăo (4), de modo que o hamil toniano que descreve a fase nemática do cristal líquido pode ser descrito por:

$$
H=-\frac{1}{2} \sum_{i, j} V_{i}, j Q_{i}{ }^{0} Q_{j}{ }^{0}-\Omega \sum_{i}\left(Q_{i}{ }^{1}-Q_{i}{ }^{\circ}\right)
$$

\section{CONCLUSÃO}

o hamiltoniano quadrupolar obtido para a fase nemática de um cristal líquido definido na equaçăo (10) acima pode ser estudado por meio de técnicas padröes de mecânica estatística. Por exemplo, usando-se técnicas de funçăo de Green as equações de movimento resultantes podem ser desacopladas ao longo de um processo autoconsistente através do uso do conceito de médias acumulantes. Os resultados obtidos devem, assim, satisfazer todas as identidades relevantes para um sistema de partículas com spins 1.

\section{REFERÊNCIAS}

1. MAIER, W., SAUPE, A., Z. Naturforschg. 14a, 882 (1959); ibdem 15 a , $287(1960)$.

2. LIN LEI, Phys. Rev. Letters 43, 1604 (1979).

3. TAREEVA, E. E., Soviet. Phys. Dkl. 22, 256 (1977).

4. SANTOS, V. H. F., LAUCK, L., Mol. Crys. and Liq. Crys. 72, 43 $(1981)$.

Recebido em janeiro,1991; aceito em março de 1991. 
